\title{
Stability and superstability of ternary homomorphisms and ternary derivations on ternary quasi-Banach algebras
}

\author{
Mostafa Osbouei ${ }^{1}$, Madjid Eshaghi Gordji ${ }^{2 *}$, Ali Ebadian ${ }^{3}$, Gholamreza Asgari $^{4}$ and Hassan Azadi Kenary ${ }^{5}$
}

\author{
* Correspondence: madjid. \\ eshaghi@gmail.com \\ ²Department of Mathematics, \\ Semnan University, Semnan, Iran \\ Full list of author information is \\ available at the end of the article
}

\begin{abstract}
In this article, we investigate the generalized Hyers-Ulam-Rassias stability, Isac-Rassias type stability and superstability of ternary homomorphisms and ternary derivations associated to the generalized $m$-variables Cauchy-Jensen functional equation

$$
\sum_{i=1}^{m} f\left(x_{i}\right)-\frac{1}{2 m}\left[\sum_{i=1}^{m} f\left(m x_{i}+\sum_{j=1, j \neq i}^{m} x_{j}\right)+f\left(\sum_{i=1}^{m} x_{i}\right)\right]=0
$$
\end{abstract}

for a fixed positive integer $m$ with $m \geq 3$ on ternary quasi-Banach algebras.

2010 Mathematics Subject Classification: 39B82; 39B52.

Keywords: superstability, Hyers-Ulam-Rassias stability, Isac-Rassias-type stability, ternary algebra, homomorphism, derivation, quasi-Banach space

\section{Introduction}

A functional equation $(\xi)$ is stable if any function $g$ satisfying the equation $(\xi)$ approximately is near to a true solution of $(\xi)$. A functional equation $(\xi)$ is superstable if any function $g$ satisfying the equation $(\xi)$ approximately is a true solution of $(\xi)$.

It is of interest to consider the concept of stability for a functional equation arising when we replace the functional equation by an inequality which acts as a perturbation of the equation.

The first stability problem was raised by Ulam [1] during his talk at the University of Wisconsin in 1940. The stability question of functional equations is that how do the solutions of the inequality differ from those of the given functional equation? If the answer is affirmative, we would say that the equation is stable.

In 1941, Hyers [2] gave a first affirmative answer to the question of Ulam for Banach spaces. Let $f: E \rightarrow E^{\prime}$ be a mapping between Banach spaces such that

$$
\|f(x+y)-f(x)-f(y)\| \leq \delta
$$

for all $x, y \in E$, and for some $\delta>0$. Then there exists a unique additive mapping $T$ : $E \rightarrow E^{\prime}$ such that

$$
\|f(x)-T(x)\| \leq \delta
$$

\section{Springer}

(c) 2012 Osbouei et al. ; licensee Springer. This is an open access article distributed under the terms of the Creative Commons Attribution License (http://creativecommons.org/licenses/by/2.0), which permits unrestricted use, distribution, and reproduction in any medium, provided the original work is properly cited. 
for all $x \in E$. Moreover if $f(t x)$ is continuous in $t \in \mathbb{R}$ for each fixed $x \in E$, then $T$ is linear. Aoki [3] and Bourgin [4] considered the stability problem with unbounded Cauchy differences. In 1978, Rassias [5] provided a generalization of Hyers' theorem by proving the existence of unique linear mappings near approximate additive mappings. It was shown by Gajda [6], as well as by Rassias and Šemrl [7] that one cannot prove a stability theorem of the additive equation for a specific function. Găvruta [8] obtained generalized result of Rassias' theorem which allows the Cauchy difference to be controlled by a general unbounded function.

Bourgin [4] is the first mathematician dealing with stability of (ring) ho-momorphism $f(x y)=f(x) f(y)$. The topic of approximate homomorphisms and approximate derivations was studied by a number of mathematicians (see [9-13], and references therein).

We refer the readers to $[2,5-8,11-51]$ and references therein for more detailed results on the stability problems of various functional equations.

We note that a quasi-norm is a real-valued function on a vector space $X$ satisfying the following properties:

(1) $\|x\| \geq 0$ for all $x \in X$ and $\|x\|=0$ if and only if $x=0$.

(2) $\|\lambda . x\|=|\lambda|$. $\|x\|$ for all $\lambda \in \mathbb{R}$ and all $x \in X$.

(3) There is a constant $K \geq 1$ such that $\|x+y\| \leq K(\|x\|+\|y\|)$ for all $x, y \in X$. The pair $(X,\|\|$.$) is called a quasi-normed space if II.II is a quasi-norm on X$. A quasiBanach space is a complete quasi-normed space. A quasi-norm II.II is called a $p$-norm $(0 \leq p \leq 1)$ if

$$
\|x+y\|^{p} \leq\|x\|^{p}+\|y\|^{p}
$$

for all $x, y \in X$. In this case, a quasi-Banach space is called a $p$-Banach space.

Ternary algebraic operations were considered in the 19th century by several mathematicians such as Cayley [52] who introduced the notion of cubic matrix which in turn was generalized by Kapranov et al. [39]. There are some applications, although still hypothetical, in the fractional quantum Hall effect, the nonstandard statistics, supersymmetric theory, and Yang-Baxter equation. The comments on physical applications of ternary structures can be found in [37,39,40,43,44,52-59].

Let $A$ be a linear space over a complex field equipped with a mapping []: $A^{3}=A \times A$ $\times A \rightarrow A$ with $(x, y, z) \mapsto[x, y, z]$ that is linear in variables $x, y, z$ and satisfies the associative identity $[[x, y, z], u, v]=[x,[y, z, u], v]=[x, y,[z, u, v]]$ for all $x, y, z, u, v$ in $A$. The pair $(A,[])$ is called a ternary algebra.

Assume that $A$ is a ternary algebra. We say $A$ has a unit if there exist an element $e \in$ $A$ such that $[e, e, a]=[e a e]=[a, e, e]=a$ for all $a \in A$.

Let $A$ be a ternary algebra and let $(A, \| . I I)$ be a quasi-Banach space ( $p$-Banach space) (with constant $K \geq 1$ ). Then $A$ is called a ternary quasi-Banach algebra (ternary $p$ Banach algebra) if $\|[x, y, z]\| \leq K\|x\|\|y\|\|z\|$ for all $x, y, z \in A$.

Let $\mathcal{A}$ and $\mathcal{B}$ be ternary algebras. A $\mathbb{C}$-linear mapping $H: \mathcal{A} \rightarrow \mathcal{B}$ is called a ternary homomorphism if

$$
H([a b c])=[H(a) H(b) H(c)]
$$

for all $a, b, c \in \mathcal{A}$. A $\mathbb{C}$-linear mapping $\delta: \mathcal{A} \rightarrow \mathcal{A}$ is called a ternary derivation if

$$
\delta([a b c])=[\delta(a) b c]+[a \delta(b) c]+[a b \delta(c)]
$$


for all $a, b, c \in \mathcal{A}$ (see $[25-31,46,60])$.

Recently, Ebadian and et al. [61] investigated the solution and stability of functional equation

$$
\sum_{i=1}^{m} f\left(m x_{i}+\sum_{j=1, j \neq i}^{m} x_{j}\right)+f\left(\sum_{i=1}^{m} x_{i}\right)=2 m \sum_{i=1}^{m} f\left(x_{i}\right)
$$

for a fixed positive integer $m$ with $m \geq 2$ in quasi-Banach spaces. In this paper, we establish the generalized Hyers-Ulam-Rassias stability of ternary homomorphisms and ternary derivations on ternary quasi-Banach algebras. Moreover, by using the main theorems, we prove the superstability of ternary homomorphisms and ternary derivations on ternary quasi Banach algebras.

Throughout this article, we assume that $A$ is a ternary quasi-Banach algebra with quasi-norm $\|.\|_{A}$ and $B$ is a ternary $p$-Banach algebra with quasi-norm $\|.\|_{B}$

\section{Ternary homomorphisms}

From now on, we assume that $m, n_{0} \in \mathbb{N}$ are positive integers $m \geq 3$, and suppose that $\mathbb{T}_{\frac{1}{n_{0}}}^{1}:=\left\{e^{i \theta} ; 0 \leq \theta \leq \frac{2 \pi}{n_{o}}\right\}$. Moreover, we will use the following abbreviation for a given mapping $f: A \rightarrow B$ :

$$
\begin{gathered}
D_{\mu} f\left(x_{1}, x_{2}, \ldots, x_{m}, a, b, c, u\right):=\sum_{i=1}^{m} f\left(m x_{i}+\sum_{j=1, j \neq i}^{m} x_{j}\right)+f\left(\sum_{i=1}^{m} x_{i}\right)-2 m \sum_{i=1}^{m} f\left(x_{i}\right) \\
+f([a b c])-[f(a) f(b) f(c)]+f(\mu u)-\mu f(u)
\end{gathered}
$$

for all $a, b, c, u, x_{1}, x_{2}, \ldots, x_{m} \in A$ and all $\mu \in \mathbb{T}_{\frac{1}{n_{o}}}^{1}$

Theorem 2.1. Let $\phi: A \underbrace{\times \cdots \times A}_{m+4-\text { times }} \rightarrow[0, \infty)$ be a function satisfying

$$
\Psi(x)=\sum_{i=1}^{\infty}\left(\frac{1}{m}\right)^{i p}\left(\phi\left(m^{i-1} x, 0, \ldots, 0\right)\right)^{p}<\infty
$$

for all $x \in A$, and

$$
\lim _{n \rightarrow \infty} \frac{1}{m^{n}} \phi\left(m^{n} x_{1}, \ldots, m^{n} x_{m}, m^{n} a, m^{n} b, m^{n} c, m^{n} u\right)=0
$$

for all $u, a, b, c, x_{j} \in A(1 \leq j \leq m)$. Let $f: A \rightarrow B$ be a mapping such that $f(0)=0$ and that

$$
\left\|D_{\mu} f\left(x_{1}, \ldots, x_{m}, a, b, c, u\right)\right\| \leq \phi\left(x_{1}, \ldots, x_{m}, a, b, c, u\right)
$$

for all $u, a, b, c, x_{j} \in A(1 \leq j \leq m)$ and all $\mu \in \mathbb{T}_{\frac{1}{n_{0}}}^{1}$. Then there exists a unique ternary homomorphism $T: A \rightarrow B$ such that inequality

$$
\|f(x)-T(x)\| \leq[\Psi(x)]^{\frac{1}{p}}
$$

for all $x \in A$. 
Proof: Putting $\mu=1, a=b=c=u=0$ in (2.2), then we have

$$
\left\|D_{1} f\left(x_{1}, \ldots, x_{m}, 0,0,0,0\right)\right\| \leq \phi\left(x_{1}, \ldots, x_{m}, 0,0,0,0\right)
$$

for all $x_{1}, x_{2}, \ldots, x_{m} \in A$. By using the Theorem 2.2 of [61], the limit

$$
\lim _{n \rightarrow \infty} \frac{1}{m^{n}} f\left(m^{n} x\right)
$$

exists for all $x \in A$ and the mapping

$$
T(x):=\lim _{n \rightarrow \infty} \frac{1}{m^{n}} f\left(m^{n} x\right) \quad(x \in A)
$$

is a unique additive function which satisfies (2.3). Moreover, one can show that $T(x)=\frac{1}{m^{n}} T\left(m^{n} x\right)=\frac{1}{m^{2 n}} T\left(m^{2 n} x\right)$ for all $x \in A$. Putting $a=b=c=x_{1}=x_{2}=\ldots=x_{m}=$ 0 in $(2.2)$ to get

$$
\|f(\mu u)-\mu f(u)\|=\left\|D_{\mu} f(0,0, \ldots, 0, u)\right\| \leq \phi(0,0, \ldots, 0, u)
$$

for all $u \in A$ and all $\mu \in \mathbb{T}^{1} \frac{1}{n_{o}}$. Then by definition of $T$ and (2.1), we have

$$
\|T(\mu u)-\mu T(u)\|=\lim _{n \rightarrow \infty} \frac{1}{m^{n}}\left\|f\left(m^{n} \mu u\right)-u f\left(m^{n} u\right)\right\| \leq \lim _{n \rightarrow \infty} \frac{1}{m^{n}} \phi\left(0,0, \ldots, 0, m^{n} u\right)=0
$$

for all $u \in A$ and all $\mu \in \mathbb{T}_{\frac{1}{n_{o}}}^{1}$. This means that

$$
T(\mu u)=\mu T(u)
$$

for all $u \in A$ and all $\mu \in \mathbb{T}^{1} \frac{1}{n_{0}}$. By the same reasoning as that in the proof of Theorem 2.1 of [19], one can show that $T: A \rightarrow B$ is $\mathbb{C}$-linear. On the other hand, by putting $u=x_{1}=x_{2}=\ldots=x_{m}=0$ in (2.2), we have

$$
\|f([a b c])-[f(a) f(b) f(c)]\| \leq \phi(0,0, \ldots, 0, a, b, c, 0)
$$

for all $a, b, c \in A$. It follows that

$$
\begin{gathered}
\|T([a b c])-[T(a) T(b) T(c)]\|=\left\|\frac{1}{m^{2 n}} T\left(\left[m^{2 n} a b c\right]\right)-\left[T(a) \frac{1}{m^{n}} T\left(m^{n} b\right) \frac{1}{m^{n}} T\left(m^{n} c\right)\right]\right\| \\
=\lim _{n \rightarrow \infty}\left\|\frac{1}{m^{3} n} f\left(\left[\left(m^{n} a\right)\left(m^{n} b\right)\left(m^{n} c\right)\right]\right)-\left[\left(\frac{1}{m^{n}} f\left(m^{n} a\right)\right)\left(\frac{1}{m^{n}} f\left(m^{n} b\right)\right)\left(\frac{1}{m^{n}} f\left(m^{n} c\right)\right)\right]\right\| \\
=\lim _{n \rightarrow \infty} \frac{1}{m^{3} n}\left\|f\left(\left[\left(m^{n} a\right)\left(m^{n} b\right)\left(m^{n} c\right)\right]\right)-\left[\left(f\left(m^{n} a\right)\right)\left(f\left(m^{n} b\right)\right)\left(f\left(m^{n} c\right)\right)\right]\right\| \\
\leq \lim _{n \rightarrow \infty} \frac{1}{m^{3} n} \phi\left(0,0, \ldots 0, m^{n} a, m^{n} b, m^{n} c, 0\right) \\
=0
\end{gathered}
$$

for all $a, b, c \in A$. This means that $T: A \rightarrow B$ is a ternary homomorphism. The uniqueness of $T$ follows from Theorem 2.2 of [61].

Corollary 2.2. Let $\theta, r, r_{j}(1 \leq j \leq m)$ be non-negative real numbers such that $0<r, r_{j}$ $<1$. Suppose that a mapping $f: A \rightarrow B$ with $f(0)=0$ satisfies the inequality 


$$
\left\|D_{\mu} f\left(x_{1}, \ldots, x_{m}, a, b, c, u\right)\right\|_{B} \leq \theta \sum_{j=1}^{m}\left\|x_{j}\right\|_{A}^{r_{j}}+\|a\|_{A}^{r}+\|b\|_{A}^{r}+\|c\|_{A}^{r}+\|u\|_{A}^{r}
$$

for all $a, b, c, u, x_{1}, x_{1}, \ldots, x_{m} \in A$ and all $\mu \in \mathbb{T}_{\frac{1}{n_{0}}}^{1}$. Then there exists a unique ternary homomorphism $T: A \rightarrow B$ such that

$$
\|f(x)-T(x)\|_{B} \leq \frac{\theta\|x\|^{r_{1}}}{m^{r_{1}}}\left\{\frac{m^{\left(1-r_{1}\right) p}}{m^{\left(1-r_{1}\right) p}-1}\right\}^{\frac{1}{p}}
$$

for all $x \in A$

Proof: It follows from Theorem 2.1 by putting

$$
\phi\left(x_{1}, \ldots, x_{m}, a, b, c, u\right)=\theta \sum_{j=1}^{m}\left\|x_{j}\right\|_{A}^{r_{j}}+\|a\|_{A}^{r}+\|b\|_{A}^{r}+\|c\|_{A}^{r}+\|u\|_{A}^{r}
$$

for all $a, b, c, u, x_{1}, x_{1}, \ldots, x_{m} \in A$.

Now, we investigate the Hyers-Ulam type stability of ternary homomor-phisms on ternary quasi Banach algebras as follows.

Corollary 2.3. Let $\theta$ be non-negative real number. Suppose that a mapping $f: A \rightarrow B$ with $f(0)=0$ satisfies the inequality

$$
\left\|D_{\mu} f\left(x_{1}, \ldots, x_{m}, a, b, c, u\right)\right\|_{B} \leq \theta
$$

for all $a, b, c, u, x_{1}, x_{1}, \ldots, x_{m} \in A$ and all $\mu \in \mathbb{T}_{\frac{1}{n_{0}}}^{1}$. Then there exists a unique ternary homomorphism $T: A \rightarrow B$ such that

$$
\|f(x)-T(x)\|_{B} \leq \theta\left\{\frac{1}{m^{p}-1}\right\}^{\frac{1}{p}}
$$

for all $x \in A$.

Proof. It follows from Theorem 2.1, by putting

$$
\phi\left(x_{1}, x_{2}, \ldots x_{m}, a, b, c, u\right):=\theta
$$

for all $u, a, b, c, x_{1}, x_{2}, \ldots, x_{m} \in A$.

Isac and Rassias [38] generalized the Hyers' theorem by introducing a mapping $\psi: \mathbb{R}$ $+\rightarrow \mathbb{R}^{+}$subject to the conditions:
1) $\lim _{t \rightarrow \infty} \frac{\psi(t)}{t}=0$,
2) $\psi(t s) \leq \psi(t) \psi(s) ; \quad s, t>0$,
3) $\psi(t)<t ; \quad t>1$.

These stability results can be applied in stochastic analysis [38], financial and actuarial mathematics, as well as in psychology and sociology. The following corollary is Isac-Rassias type stability of ternary homomorphisms on ternary quasi-Banach algebras. 
Corollary 2.4. Let $\psi: \mathbb{R}^{+} \rightarrow \mathbb{R}^{+}$be a mapping such that

$$
\begin{gathered}
\lim _{t \rightarrow \infty} \frac{\psi(t)}{t}=0 \\
\psi(t s) \leq \psi(t) \psi(s) \quad s, t>0, \\
\psi(t)<t \quad t>1 .
\end{gathered}
$$

Let $\theta, r, r_{j}(1 \leq j \leq m)$ be non-negative real numbers. Let $f: A \rightarrow B$ be a mapping such that $f(0)=0$ and that

$$
\left\|D_{\mu} f\left(x_{1}, \ldots, x_{m}, a, b, c, u\right)\right\|_{B} \leq \theta\left(\sum_{j=1}^{m} \psi\left(\left\|x_{j}\right\|_{A}\right)+\psi\left(\|a\|_{A}\right)+\psi\left(\|b\|_{A}\right)+\psi\left(\|c\|_{A}\right)+\psi\left(\|u\|_{A}\right)\right)
$$

for all $u, a, b, c, x_{1}, x_{2}, \ldots, x_{m} \in A$. Then there exists a unique ternary homo-morphism $T: A \rightarrow B$ such that

$$
\|f(x)-T(x)\|_{B} \leq k \theta \psi\left(m^{-1}\right) \psi(\|x\|)
$$

for all $x \in A$, where $k=\frac{\psi(m)}{m-\psi(m)}$.

Proof: The proof follows from Theorem 2.1 by taking

$$
\phi\left(x_{1}, \ldots, x_{m}, a, b, c, u\right):=\theta\left(\sum_{j=i}^{m} \psi\left(\left\|x_{j}\right\|_{A}\right)+\psi\left(\|a\|_{A}\right)+\psi\left(\|b\|_{A}\right)+\psi\left(\|c\|_{A}\right)+\psi\left(\|u\|_{A}\right)\right)
$$

for all $u, a, b, c, x_{1}, x_{2}, \ldots, x_{m} \in A$.

Moreover, we have the superstability of ternary homomorphisms on ternary quasi Banach algebras as follows.

Corollary 2.5. Let $\theta, r, r_{j}(1 \leq j \leq m)$ be non-negative real numbers such that $0<r, r_{j}$ $<1$. Suppose that a mapping $f: A \rightarrow B$ with $f(0)=0$ satisfies the inequality

$$
\left\|D_{\mu} f\left(x_{1}, \ldots, x_{m}, a, b, c, u\right)\right\|_{B} \leq \theta\left(\prod_{j=1}^{m}\left\|x_{j}\right\|_{A}^{r_{j}}\right)\|a\|_{A}^{r}\|b\|_{A}^{r}\|c\|_{A}^{r}\|u\|_{A}^{r}
$$

for all $a, b, c, u, x_{1}, x_{1}, \ldots, x_{m} \in A$ and all $\mu \in \mathbb{T}_{\frac{1}{n_{o}}}^{1}$. Then $f: A \rightarrow B$ is a ternary homomorphism.

Proof: Putting

$$
\phi\left(x_{1}, \ldots, x_{m}, a, b, c, u\right):=\theta\left(\prod_{j=1}^{m}\left\|x_{j}\right\|_{A}^{r_{j}}\right)\|a\|_{A}^{r}\|b\|_{A}^{r}\|c\|_{A}^{r}\|u\|_{A}^{r}
$$

for all $a, b, c, u, x_{1}, x_{1}, \ldots, x_{m} \in A$. Then we have $\varphi(x, 0,0, \ldots, 0)=0$. By Theorem 2.1, there exists a unique ternary homomorphism $T: A \rightarrow B$ such that

$$
\|f(x)-T(x)\|_{B} \leq[\Psi(x)]^{\frac{1}{p}}=0
$$

for all $x \in A$. This means that $f(x)=T(x)$ for all $x \in A$. Hence $f: A \rightarrow B$ is a ternary homomorphism. 


\section{Ternary derivations}

In this section, we use the following abbreviation for a given mapping $f: A \rightarrow A$ :

$$
\begin{aligned}
\Delta_{\mu} f\left(x_{1}, x_{2}, \ldots, x_{m}, a, b, c, u\right) & :=\sum_{i=1}^{m} f\left(m x_{i}+\sum_{j=1, j \neq i}^{m} x_{j}\right)+f\left(\sum_{i=1}^{m} x_{i}\right)-2 m \sum_{i=1}^{m} f\left(x_{i}\right) \\
+f([a b c])-[f(a) b c] & -[a f(b) c]-[a b f(c)]+f(\mu u)-\mu f(u)
\end{aligned}
$$

for all $a, b, c, u, x_{1}, x_{2}, \ldots, x_{m} \in A$ and all $\mu \in \mathbb{T}_{\frac{1}{n_{o}}}^{1}$.

Theorem 3.1. Let $\phi: A \underbrace{\times \cdots \times A}_{m+4-\text { times }} \rightarrow[0, \infty)$ be a function satisfying

$$
\Psi(x)=\sum_{i=1}^{\infty}\left(\frac{1}{m}\right)^{i p}\left(\phi\left(m^{i-1} x, 0, \ldots, 0\right)\right)^{p}<\infty
$$

for all $x \in A$, and

$$
\lim _{n \rightarrow \infty} \frac{1}{m^{n}} \phi\left(m^{n} x_{1}, \ldots, m^{n} x_{m}, m^{n} a, m^{n} b, m^{n} c, m^{n} u\right)=0
$$

for all $u, a, b, c, x_{j} \in A(1 \leq j \leq m)$. Let $f: A \rightarrow B$ be a mapping such that $f(0)=0$ and that

$$
\left\|\Delta_{\mu} f\left(x_{1}, \ldots, x_{m}, a, b, c, u\right)\right\| \leq \phi\left(x_{1}, \ldots, x_{m}, a, b, c, u\right)
$$

for all $u, a, b, c, x_{j} \in A(1 \leq j \leq m)$ and all $\mu \in \mathbb{T}_{\frac{1}{n_{o}}}^{1}$. Then there exists a unique ternary derivation $D: A \rightarrow B$ such that

$$
\|f(x)-D(x)\| \leq[\Psi(x)]^{\frac{1}{p}}
$$

for all $x \in A$.

Proof: By using the same technique of proving Theorem 2.1, the limit

$$
\lim _{n \rightarrow \infty} \frac{1}{m^{n}} f\left(m^{n} x\right)
$$

exists for all $x \in A$ and the mapping

$$
D(x):=\lim _{n \rightarrow \infty} \frac{1}{m^{n}} f\left(m^{n} x\right) \quad(x \in A)
$$

is a unique $\mathbb{C}$-linear function which satisfies (2.3). On the other hand, by putting $u=$ $x_{1}=x_{2}=\cdots=x_{m}=0$ in (3.1), we have

$$
\|f([a b c])-[f(a) b c]-[a f(b) c]-[a b f(c)]\| \leq \phi(0,0, \ldots, 0, a, b, c, 0)
$$


for all $a, b, c \in A$. It follows that

$$
\begin{gathered}
\|D([a b c])-[D(a) b c]-[a D(b) c]-[a b D(c)]\| \\
=\| \frac{1}{m^{2 n}} D\left(\left[m^{2 n} a b c\right]\right)-\left[D(a) \frac{1}{m^{n}}\left(m^{n} b\right) \frac{1}{m^{n}}\left(m^{n} c\right)\right] \\
-\left[\frac{1}{m^{n}}\left(m^{n} a\right) D(b) \frac{1}{m^{n}}\left(m^{n} c\right)\right]-\left[\frac{1}{m^{n}} D\left(m^{n} a\right) \frac{1}{m^{n}}\left(m^{n} b\right) D(c)\right] \| \\
=\lim _{n \rightarrow \infty} \| \frac{1}{m^{3} n} f\left(\left[\left(m^{n} a\right)\left(m^{n} b\right)\left(m^{n} c\right)\right]\right)-\left[\left(\frac{1}{m^{n}} f\left(m^{n} a\right)\right)\left(\frac{1}{m^{n}}\left(m^{n} b\right)\right)\left(\frac{1}{m^{n}}\left(m^{n} c\right)\right)\right] \\
-\left[\left(\frac{1}{m^{n}}\left(m^{n} a\right)\right)\left(\frac{1}{m^{n}} f\left(m^{n} b\right)\right)\left(\frac{1}{m^{n}}\left(m^{n} c\right)\right)\right]-\left[\left(\frac{1}{m^{n}}\left(m^{n} a\right)\right)\left(\frac{1}{m^{n}}\left(m^{n} b\right)\right)\left(\frac{1}{m^{n}} f\left(m^{n} c\right)\right)\right] \| \\
=\lim _{n \rightarrow \infty} \frac{1}{m^{3} n} \| f\left(\left[\left(m^{n} a\right)\left(m^{n} b\right)\left(m^{n} c\right)\right]\right)-\left[\left(f\left(m^{n} a\right)\right)\left(\left(m^{n} b\right)\right)\left(\left(m^{n} c\right)\right)\right] \\
-\left[\left(\left(m^{n} a\right)\right)\left(f\left(m^{n} b\right)\right)\left(\left(m^{n} c\right)\right)\right]-\left[\left(\left(m^{n} a\right)\right)\left(\left(m^{n} b\right)\right)\left(f\left(m^{n} c\right)\right)\right] \| \\
\leq \lim _{n \rightarrow \infty} \frac{1}{m^{3} n} \phi\left(0,0, \ldots, 0, m^{n} a, m^{n} b, m^{n} c, 0\right) \\
=0
\end{gathered}
$$

for all $a, b, c \in A$. This means that $D: A \rightarrow B$ is a ternary derivation.

Corollary 3.2. Let $\theta, r, r_{j}(1 \leq j \leq m)$ be non-negative real numbers such that $0<r, r_{j}$ $<1$. Suppose that a mapping $f: A \rightarrow B$ with $f(0)=0$ satisfies the inequality

$$
\left\|\Delta_{\mu} f\left(x_{1}, \ldots, x_{m}, a, b, c, u\right)\right\|_{A} \leq \theta\left(\sum_{j=1}^{m}\left\|x_{j}\right\|_{A}^{r_{j}}+\|a\|_{A}^{r}+\|b\|_{A}^{r}+\|c\|_{A}^{r}+\|u\|_{A}^{r}\right.
$$

for all $a, b, c, u, x_{1}, x_{1}, \ldots, x_{m} \in A$ and all $\mu \in \mathbb{T}_{\frac{1}{n_{o}}}^{1}$. Then there exists a unique ternary derivation $D: A \rightarrow B$ such that

$$
\|f(x)-D(x)\|_{A} \leq \frac{\theta\|x\|^{r_{1}}}{m^{r_{1}}}\left\{\frac{m^{\left(1-r_{1}\right) p}}{m^{\left(1-r_{1}\right) p}-1}\right\}^{\frac{1}{p}}
$$

for all $x \in A$.

Proof: It follows from Theorem 3.1 by putting

$$
\phi\left(x_{1}, \ldots, x_{m}, a, b, c, u\right)=\theta \sum_{j=1}^{m}\left\|x_{j}\right\|_{A}^{r_{j}}+\|a\|_{A}^{r}+\|b\|_{A}^{r}+\|c\|_{A}^{r}+\|u\|_{A}^{r}
$$

for all $a, b, c, u, x_{1}, x_{1}, \ldots, x_{m} \in A$.

We have the Hyers-Ulam type stability of ternary derivations on ternary quasi Banach algebras as follows.

Corollary 3.3. Let $\theta$ be non-negative real number. Suppose that a mapping $f: A \rightarrow B$ with $f(0)=0$ satisfies the inequality

$$
\left\|\Delta_{\mu} f\left(x_{1}, \ldots, x_{m}, a, b, c, u\right)\right\|_{A} \leq \theta
$$

for all $a, b, c, u, x_{1}, x_{1}, \ldots, x_{m} \in A$ and all $\mu \in \mathbb{T}_{\frac{1}{n_{0}}}^{1}$. Then there exists a unique ternary derivation $D: A \rightarrow B$ such that

$$
\|f(x)-D(x)\|_{A} \leq \theta\left\{\frac{1}{m^{p}-1}\right\}^{\frac{1}{p}}
$$


for all $x \in A$.

Proof. It follows from Theorem 3.1, by putting

$$
\phi\left(x_{1}, x_{2}, \ldots, x_{m}, a, b, c, u\right):=\theta
$$

for all $u, a, b, c, x_{1}, x_{2}, \ldots, x_{m} \in A$.

By using the same technique of proving Corollary 2.4, we can prove the Isac-Rassias type stability of ternary derivations on ternary quasi-Banach algebras as follows.

Corollary 3.4. Let $\psi: \mathbb{R}^{+} \rightarrow \mathbb{R}^{+}$be a mapping such that

$$
\begin{gathered}
\lim _{t \rightarrow \infty} \frac{\psi(t)}{t}=0 \\
\psi(t s) \leq \psi(t) \psi(s) \quad s, t>0, \\
\psi(t)<t \quad t>1 .
\end{gathered}
$$

Let $\theta, r, r_{j}(1 \leq j \leq m)$ be non-negative real numbers. Let $f: A \rightarrow A$ be a mapping such that $f(0)=0$ and that

$$
\left\|\Delta_{\mu} f\left(x_{1}, \ldots, x_{m}, a, b, c, u\right)\right\|_{A} \leq \theta\left(\sum_{j=1}^{m} \psi\left(\left\|x_{j}\right\|_{A}\right)+\psi\left(\|a\|_{A}\right)+\psi\left(\|b\|_{A}\right)+\psi\left(\|c\|_{A}\right)+\psi\left(\|u\|_{A}\right)\right)
$$

for all $u, a, b, c, x_{1}, x_{2}, \ldots, x_{m} \in A$. Then there exists a unique ternary derivation $D: A$ $\rightarrow$ A such that

$$
\|f(x)-D(x)\|_{A} \leq k \theta \psi\left(m^{-1}\right) \psi(\|x\|)
$$

for all $x \in A$, where $k=\frac{\psi(m)}{m-\psi(m)}$.

Similar to Corollary 2.5, we can prove the superstability of ternary derivations on ternary quasi-Banach algebras as follows.

Corollary 3.5. Let $\theta, r, r_{j}(1 \leq j \leq m)$ be non-negative real numbers such that $0<r, r_{j}$ $<1$. Let $f: A \rightarrow A$ be a mapping such that $f(0)=0$ and that

$$
\left\|\Delta_{\mu} f\left(x_{1}, \ldots, x_{m}, a, b, c, u\right)\right\|_{A} \leq \theta\left(\prod_{j=1}^{m}\left\|x_{j}\right\|_{A}^{r_{j}}\right) \quad\|a\|_{A}^{r}\|b\|_{A}^{r}\|c\|_{A}^{r}\|u\|_{A}^{r}
$$

for all $a, b, c, u, x_{1}, x_{1}, \ldots, x_{m} \in A$ and all $\mu \in \mathbb{T}_{\frac{1}{n_{o}}}^{1}$. Then $f: A \rightarrow A$ is a ternary derivation.

\section{Author details}

${ }^{1}$ Department of Mathematics, Islamic Azad University, Saravan Branch, Saravan, Iran ${ }^{2}$ Department of Mathematics, Semnan University, Semnan, Iran ${ }^{3}$ Department of Mathematics, Payame Noor University, P.O. Box 19395-3697, Tehran, Iran ${ }^{4}$ Department of Mathematics, Islamic Azad University, Aligoudarz Branch, Aligoudarz, Iran ${ }^{5}$ Department of Mathematics, Yasouj University, Yasouj 75914-353, Iran

Authors' contributions

All authors read and approved the final manuscript.

Competing interests

The authors declare that they have no competing interests. 


\section{References}

1. Ulam, SM: A Collection of the Mathematical Problems. Interscience Publishers, New York (1960)

2. Hyers, DH: On the stability of the linear functional equation. Proc Natl Acad Sci. 27, 222-224 (1941). doi:10.1073/ pnas.27.4.222

3. Aoki, T: On the stability of the linear transformation in Banach spaces. J Math Soc Jpn. 2, 64-66 (1950). doi:10.2969/ jmsj/00210064

4. Bourgin, DG: Classes of transformations and bordering transformations. Bull Am Math Soc. 57, 223-237 (1951). doi:10.1090/S0002-9904-1951-09511-7

5. Rassias, ThM: On the stability of the linear mapping in Banach spaces. Proc Am Math Soc. 72, 297-300 (1978). doi:10.1090/S0002-9939-1978-0507327-1

6. Gajda, Z: On stability of additive mappings. Int J Math Math Sci. 14, 431-434 (1991). doi:10.1155/S016117129100056X

7. Rassias, ThM, Šemrl, P: On the behavior of mappings which do not satisfy Hyers-Ulam stability. Proc Am Math Soc. 114, 989-993 (1992). doi:10.1090/S0002-9939-1992-1059634-1

8. Găvruta, P: A generalization of the Hyers-Ulam-Rassias stability of approximately additive mappings. J Math Anal Appl. 184, 431-436 (1994). doi:10.1006/jmaa.1994.1211

9. Badora, R: On approximate ring homomorphisms. J Math Anal Appl. 276, 589-597 (2002). doi:10.1016/S0022-247X(02) 00293-7

10. Badora, R: On approximate derivations. Math Inequal Appl. 9, 167-173 (2006)

11. Hyers, DH, Rassias, ThM: Approximate homomorphisms. Aequationes Math. 44, 125-153 (1992). doi:10.1007/BF01830975

12. Miura, T, Hirasawa, G, Takahasi, SE: A perturbation of ring derivations on Banach algebras. J Math Anal Appl. 319 522-530 (2006). doi:10.1016/j.jmaa.2005.06.060

13. Park, C: Hyers-Ulam-Rassias stability of homomorphisms in quasi-Banach algebras. Bull Sci Math. 132(2):87-96 (2008). doi:10.1016/j.bulsci.2006.07.004

14. Ebadian, A, Ghobadipour, N, Eshaghi Gordji, M: A fixed point method for perturbation of bimultipliers and Jordan bimultipliers in $C^{*}$-ternary algebras. J Math Phys. 51, 103508 (2010). doi:10.1063/1.3496391

15. Ebadian, A, Ghobadipour, N, Banand Savadkouhi, M, Eshaghi Gordji, M: Stability of a mixed type cubic and quartic functional equation in non-Archimedean $\ell$-fuzzy normed spaces. Thai J Math. 9(2):225-241 (2011)

16. Ebadian, A, Ghobadipour, N, Rassias, ThM, Eshaghi Gordji, M: Functional inequalities associated with cauchy additive functional equation in non-Archimedean spaces. Discrete Dyn Nat Soc 2011 (2011). Article ID 929824

17. Ebadian, A, Ghobadipour, N, Rassias, ThM, Nikoufar, I: Stability of generalized derivations on Hilbert $C^{*}$-modules associated to a pexiderized Cuachy-Jensen type functional equation. Acta Mathematica Scintia. 32(3):1226-1238 (2012). doi:10.1016/S0252-9602(12)60094-0

18. Ebadian, A, Najati, A, Eshaghi Gordji, M: On approximate additive-quartic and quadratic-cubic functional equations in two variables on abelia groups. Results Math. 58, 39-53 (2010). doi:10.1007/s00025-010-0018-4

19. Eshaghi Gordji, M: Nearly involutions on Banach algebras: a fixed point approach. Fixed Point Theory. (in press)

20. Eshaghi Gordji, M, Bavnd Savadkouhei, M: Approximation of generalized homomor-phisms in quasi-Banach algebras. Analele Univ Ovidius Constata, Math series. 17(2):203-214 (2009)

21. Eshaghi Gordji, M, Ghobadipour, N: Nearly generalized Jordan derivations. Math Slo-vaca. 61(1):1-8 (2011). doi:10.2478/ s12175-010-0055-1

22. Eshaghi Gordji, M, Ghobadipour, N: Stability of $(a, \beta, \gamma)$-derivations on Lie $C^{*}$-algebras. Int J Geometric Methods Modern Phys. 7(7):1093-1102 (2010). doi:10.1142/S0219887810004737

23. Eshaghi Gordji, M, Rassias, JM, Ghobadipour, N: Generalized Hyers-Ulam stability of generalized ( $n, k)$-derivations. Abst Appl Anal 8 (2009). 2009, Article ID 437931

24. Eshaghi Gordji, M, Ghobadipour, N: Approximately quartic homomorphisms on Banach algebras. Word Appl Sci J. (in press)

25. Eshaghi Gordji, M: Nearly ring homomorphisms and nearly ring derivations on non-Archimedean Banach algebras. Abst Appl Anal 2010, 12 (2010). Article ID 393247

26. Eshaghi Gordji, M, Alizadeh, Z: Stability and superstability of ring homomorphisms on non-Archimedean Banach algebras. Abst Appl Anal 2011, 10 (2011). Article ID 123656

27. Eshaghi Gordji, M, Ghaemi, MB, Kaboli Gharetapeh, S, Shams, S, Ebadian, A: On the stability of J*-derivations. J Geometry Phys. 60(3):454-459 (2010). doi:10.1016/j.geomphys.2009.11.004

28. Eshaghi Gordji, M, Kaboli Gharetapeh, S, Savadkouhi, MB, Aghaei, M, Karimi, T: On cubic derivations. Int J Math Anal. 4(51):2501-2514 (2010)

29. Eshaghi Gordji, M, Karimi, T, Kaboli Gharetapeh, S: Approximately n-Jordan homo-morphisms on Banach algebras. J Ineq Appl 2009, 8 (2009). Article ID 870843

30. Eshaghi Gordji, M, Moslehian, MS: A trick for investigation of approximate derivations. Math Commun. 15(1):99-105 (2010)

31. Farokhzad, R, Hosseinioun, SAR: Perturbations of Jordan higher derivations in Banach ternary algebras: an alternative fixed point approach. Int J Nonlinear Anal Appl. 1(1):42-53 (2010)

32. Eskandani, GZ: On the Hyers-Ulam-Rassias stability of an additive functional equation in quasi-Banach spaces. J Math Anal Appl. 345, 405-409 (2008). doi:10.1016/j.jmaa.2008.03.039

33. Gavruta, P, Gavruta, L: A new method for the generalized Hyers-Ulam-Rassias stability. Int J Nonlinear Anal Appl. 1(2):11-18 (2010)

34. Gajda, Z, Ger, R: Subadditive multifunctions and Hyers-Ulam stability. General Inequalities, vol. 5. International Schriftenreiche Numer Math 80. Birkhuser, Basel-Boston, MA (1987)

35. Gruber, PM: Stability of isometries. Trans Am Math Soc. 245, 263-277 (1978)

36. Ghobadipour, N, Ebadian, A, Rassias, ThM, Eshaghi, M: A perturbation of double derivations on Banach algebras. Commun Math Anal. 11(1):51-60 (2011)

37. Haag, R, Kastler, D: An algebraic approach to quantum field theory. J Math Phys. 5, 848-861 (1964). doi:10.1063/ 1.1704187

38. Isac, G, Rassias, ThM: Stability of $\psi$-additive mappings: applications to nonlinear analysis. Int J Math Math Sci. 19, 219-228 (1996). doi:10.1155/S0161171296000324 
39. Kapranov, M, Gelfand, IM, Zelevinskii, A: Discrimininants. Resultants and Multidimensional Determinants. Birkhauser, Berlin (1994)

40. Kerner, R: The cubic chessboard. Geometry Phys Class Quant Grav. 14, A203-A225 (1997)

41. Malliavin, P: Stochastic Analysis. Springer, Berlin (1997)

42. Park, CG: Linear *-derivations on $C^{*}$-algebras. Tamsui Oxf J Math Sci. 23(2):155-171 (2007)

43. Nambu, Y: Generalized Hamiltonian mechanics. Phys Rev. D7, 2405-2412 (1973)

44. Okubo, S: Triple products and Yang-Baxter equation (I): octonional and quaternionic triple systems. J Math, Phys. 34(7):3273-3291 (1993). doi:10.1063/1.530076

45. Park, C: Homomorphisms between Lie $J C^{*}$-algebras and Cauchy-Rassias stability of Lie $J C^{*}$-algebra derivations. J Lie Theory. 15, 393-414 (2005)

46. Park, C, Eshaghi Gordji, M: Comment on "Approximate ternary Jordan derivations on Banach ternary algebras" [Bavand Savadkouhi et al. J. Math. Phys. 50, 042303 (2009). J Math Phys. 51(044102):7 (2010)

47. Rassias, JM: Solution of the Ulam stability problem for quartic mappings. Glasnik Matematicki. 34, 243-252 (1999)

48. Rassias, JM: On a new approximation of approximately linear mappings by linear mappings. Discus Math. 7, 193-196 (1985)

49. Rassias, JM: On approximation of approximately linear mappings by linear mappings. J Funct Anal. 46(1):126-130 (1982). doi:10.1016/0022-1236(82)90048-9

50. Rassias, ThM: Problem 16; 2, Report of the 27th International Symp.on Functional Equations. Aequationes Math. 39 , 292-293 (1990)

51. Rassias, ThM (eds.): Functional Equations, Inequalities and Applications. Kluwer Academic, Dordrecht (2003)

52. Cayley, A: On the 34 concomitants of the ternary cubic. Am J Math. 4(1-4):1-15 (1881)

53. Abramov, V, Kerner, R, Le Roy, B: Hypersymmetry a $Z_{3}$ graded generalization of supersymmetry. J Math Phys. 38 , 1650-1669 (1997). doi:10.1063/1.531821

54. Bazunova, N, Borowiec, A, Kerner, R: Universal differential calculus on ternary algebras. Lett Matt Phys. 67(3):195-206 (2004)

55. Bagarello, F, Morchio, G: Dynamics of mean-field spin models from basic results in abstract differential equations. J Stat Phys. 66, 849-866 (1992). doi:10.1007/BF01055705

56. Sewell, GL: Quantum Mechanics and its Emergent Macrophysics. Princeton Universtiy Press, Princeton, NJ (2002) MR1919619 (2004b:82001)

57. Takhtajan, L: On foundation of the generalized Nambu mechanics. Commun Math Phys. 160(2):295-315 (1994). doi:10.1007/BF02103278

58. Vainerman, L, Kerner, R: On special classes of n-algebras. J Math Phys. 37(5):2553-2565 (1996). doi:10.1063/1.531526

59. Zettl, H: A characterization of ternary rings of operators. Adv Math. 48, 117-143 (1983). doi:10.1016/0001-8708(83)90083$x$

60. Bavand Savadkouhi, M, Gordji, ME, Rassias, JM, Ghobadipour, N: Approximate ternary Jordan derivations on Banach ternary algebras. J Math Phys. 50(042303):9 (2009)

61. Ebadian, A, Ghobadipour, N, Eshaghi Gordji, M: On the stability of a parametric-additive functional equations in quasiBanach spaces. Abst Appl Anal 2012, 13 (2012). Art id 235359

doi:10.1186/1687-1847-2012-80

Cite this article as: Osbouei et al:: Stability and superstability of ternary homomorphisms and ternary derivations on ternary quasi-Banach algebras. Advances in Difference Equations 2012 2012:80.

\section{Submit your manuscript to a SpringerOpen ${ }^{\odot}$ journal and benefit from:}

- Convenient online submission

- Rigorous peer review

- Immediate publication on acceptance

- Open access: articles freely available online

- High visibility within the field

- Retaining the copyright to your article

Submit your next manuscript at $\gg$ springeropen.com 